\title{
A Self-Organized Plasma with Induction, Reconnection, and Injection Techniques: the SPIRIT Concept for Field Reversed Configuration Research
}

\author{
Masaaki YAMADA, Hantao JI, Stefan P. GERHARDT, Elena V. BELOVA, \\ Ronald C. DAVIDSON and David R. MIKKELSEN \\ Princeton Plasma Physics Laboratory, Princeton University, Princeton, NJ, 08543, USA
}

(Received 10 August 2006 / Accepted 25 January 2007)

\begin{abstract}
A comprehensive research concept, known as SPIRIT, is described for the investigation of the formation, stability, and sustainment of oblate field reversed configurations (FRCs). This concept, whose name stands for Self-organized Plasma with Induction, Reconnection, and Injection Techniques (SPIRIT), allows for the study of FRC stability properties on time scales much longer than the energy confinement time. Counter-helicity merging of inductively formed spheromaks is utilized to form large-flux FRCs. These FRCs are sustained by neutral beam injection with the initial aid of compact ohmic solenoids. Stability to $n=1$ tilt/shift modes is provided by plasma shaping and conducting shells. Stability to $n \geq 2$ co-interchange modes is achieved by a distribution of highenergy non-thermal ions provided by the neutral beam. The combination of plasma shaping, conducting shells, current sustainment, and the non-thermal beam component are expected to lead to a configuration with stability to all global MHD modes, a regime recently discovered through hybrid-MHD simulation using the HYM code. An experimental test of the concept, utilizing the existing Magnetic Reconnection Experiment (MRX) facility, is described. Initial experiments in MRX have confirmed the viability of the SPIRIT concept, and calculations indicate that the confinement of high-energy ions in MRX should be sufficient to test the SPIRIT concept.
\end{abstract}

(C) 2007 The Japan Society of Plasma Science and Nuclear Fusion Research

Keywords: SPIRIT, FRC (Field-Reversed Configuration), FLR effects (Finite-Larmour Radius effects), oblate FRC

DOI: $10.1585 /$ pfr.2.004

\section{Introduction}

Compact toroidal plasma configurations, such as fieldreversed configurations (FRCs), have been studied in the search for a cost-effective, high-performance, high-powerdensity reactor concept [1-5]. Since FRC configurations have the highest beta among magnetic fusion energy configurations, they offer the possibility of an advanced-fuel reactor, provided the confinement and stability is sufficiently favorable. The major scientific challenges in FRC research are the significant uncertainties in their stability and confinement properties [3]. Although high-beta FRC equilibria can be obtained relatively easily, plasma lifetimes are limited to be of the order of the energy confinement time $(<1 \mathrm{msec})$, and long-time stability properties are yet to be conclusively verified.

This paper describes an experimental concept that addresses these three critical issues by stabilizing and sustaining an FRC plasma with the use of plasma shaping, conducting shells, neutral beam injection (NBI) and a current transformer. In the SPIRIT concept, compact toroid plasmas would be generated by the merging of co- and counter-helicity spheromaks produced by inductive discharges utilizing flux cores, and the plasmas would be sus- tained by the use of neutral beam injection (NBI) and inductive transformers. Plasma shaping, conducting shells, and the NBI would provide stabilization to MHD modes. This generic idea is testable in existing oblate FRC devices, and has led to the conceptual design of a series of experiments which could be carried out in the Magnetic Reconnection Experiment (MRX) device [6]. An FRC reactor design is outside the scope of the present paper, although we note that promising FRC reactor concepts have been developed in the past [7].

This physics basis for the SPIRIT concept has been verified in numerical calculations [8-13], as well as oblate FRC experiments. Recent numerical studies have found an operation regime for an oblate FRC which is stable against all global modes. This configuration utilizes a stabilizing shell and neutral beam injection with a practically broad velocity distribution [13]. Experimentally, slow formation of an FRC plasma by the merging of spheromak plasmas has been successfully demonstrated in a number of experiments: the TS-3/4 devices [14-21] at the University of Tokyo, the Swarthmore Spheromak Experiment [22] (SSX), and MRX [23,24] at Princeton Plasma Physics Laboratory (PPPL). Sustainment of an FRC plasma by an Ohmic solenoid has been demonstrated [15]. The ex- 
perimental observation of the co-interchange instability in MRX [24] yields further motivation for the NBI system included in the SPIRIT concept.

Many of these techniques unique to the SPIRIT concept are illustrated in Fig. 1, where a schematic of a generic full-size SPIRIT experimental device is illustrated. Spheromaks are formed by a method utilizing a pair of flux-cores on each end of the device. These spheromaks, which have oppositely directed toroidal fields, are allowed to merge in the center of the machine, producing the FRC that is illustrated. The combination of the equilibrium field (EF) coils, flux-core windings, and shaping field coils is used to control the plasma shape. To stabilize $n=1$ tilt/shift modes [25-27], passively stabilizing shells are utilized. A $25 \mathrm{kV}, 60 \mathrm{~A}$ neutral beam is injected into this target plasma. The kinetic effects associated with these beam particles aids in the stabilization of co-interchange modes [9, 28-30], as well as helping to sustain the plasma pressure, and hence the diamagnetic toroidal current of the FRC. Other components of the SPIRIT concept, such as possible ohmic transformers [will be described with Fig. 10] and quadrapole stabilizers for the $n=2$ rotational mode [31], are not illustrated in this figure. It is the goal of this paper to describe the scientific baseline for the SPIRIT concept, and to provide a conceptual design for an implementation of the SPIRIT concept on the existing MRX device.

A key element of the SPIRIT program is NBI stabilization of the plasma via finite Larmor radius (FLR) effects; the target plasma must have sufficient orbit confinement of these high-energy particles for this scheme to be successful. In order to generate plasmas with proper flux levels and shaping for the NBI, FRC plasmas are generated by the counter-helicity merging of inductively formed spheromaks. This flux-core based inductive formation scheme, which uses neither conventional fast shock heating nor electrode discharges, has significant advantages for

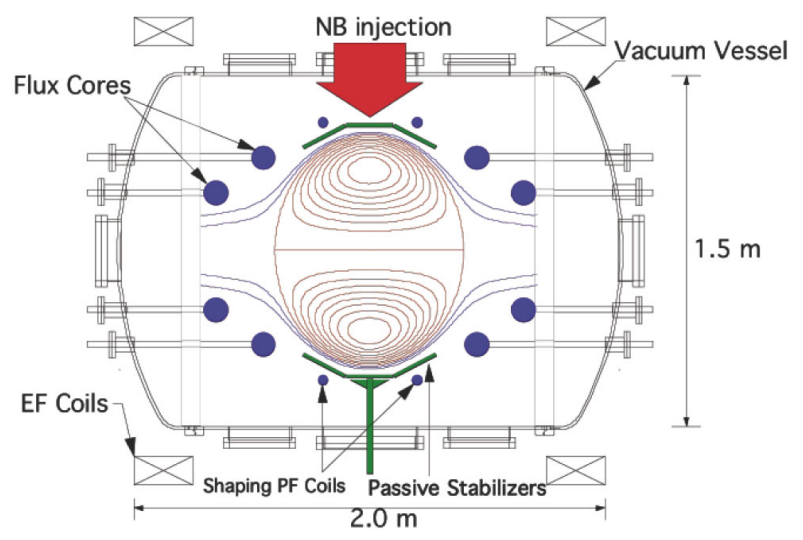

Fig. 1 Schematics of the full-sized SPIRIT device, including flux-cores, passive stabilizers, shaping coils, and neutral beam injection. the development of a compact reactor core. A series of successful spheromak merging experiments have been carried out on the devices in which FRC configurations are formed by the merging of two toroidal plasmas with opposing toroidal fields. These experiments have established the technical feasibility of this approach.

The SPIRIT concept uniquely complements other existing FRC experiments in the U.S. and elsewhere in the world. The FRC experiments at the University of Washington focus on the translation of theta-pinch formed plasmas [32], and the formation and sustainment of plasmas with the use of RMF (rotating magnetic field) techniques [33-35]. The MTF (magnetic target fusion) experiment at Los Alamos focuses on formation of FRC plasmas by traditional theta-pinch techniques, aimed at providing a high-density plasma target for further compression [36, 37]. The SSX experiment at Swarthmore College [22] and the TS-3/4 experiments at the University of Tokyo [14-21] continue to focus on the innovative formation and stability of FRC plasmas with relatively small sizes and without a neutral beam injection system. Early versions of the SPIRIT concept has been previously discussed [38,39], though this paper represents a more mature version of the concept.

The remainder of this paper is organized as follows. Recent theoretical and experimental advances relevant to the SPIRIT program are presented in Sec. 2. Experimental and theoretical scoping studies are described in Sec. 3. A more detailed description of selected aspects of the experiments in the MRX device is provided in Sec. 4. Finally, a summary is presented in Sec. 5 .

\section{Recent Scientific Advances in FRC Research}

This section provides a general overview of recent progress in FRC research. It includes both theoretical studies in Sec. 2.1 and experimental research in Sec. 2.2. This scientific understanding of FRC plasmas provides a firm foundation for the research program described in this paper.

\subsection{Recent advances in FRC theory}

One of the most important scientific issues in fieldreversed configuration (FRC) research is the stability with respect to low- $n$ (toroidal mode number) magnetohydrodynamic (MHD) modes. Significant progress in the theoretical understanding of FRC stability properties has been achieved in the past few years. According to an empirical scaling relation [40] based on experimental data for prolate FRCs, stability with respect to global MHD modes is achieved for $S^{*} / E<3-4$, where $E$ is the separatrix elongation, and the kinetic parameter $S^{*}=R_{\mathrm{S}} / \lambda_{\mathrm{i}}$, is the ratio of the separatrix radius to the ion skin depth $(S *$ is also approximately equal to $s$, the ratio of the separatrix radius to the ion gyroradius $\rho_{\mathrm{i}}$ in the external magnetic 
Table 1 Stability properties of prolate and oblate FRCs.

\begin{tabular}{|l|l|l|}
\hline Mode & Prolate $(E>1)$ & Oblate $(E<1)$ \\
\hline Internal tilt, $n=1$ & $\begin{array}{l}\text { MHD Unstable, stabilized by FLR, } \\
\text { rotation and nonlinear effects for } \\
S^{*}<20 \text { and } E \geq 5\end{array}$ & MHD Stable \\
\hline $\begin{array}{l}\text { External tilt and } \\
\text { radial shift, } n=1\end{array}$ & MHD Stable & $\begin{array}{l}\text { MHD Unstable, stabilized } \\
\text { by conducting shell }\end{array}$ \\
\hline $\begin{array}{l}\text { Co-interchange, } \\
n>1\end{array}$ & MHD Unstable, stabilized by FLR & $\begin{array}{l}\text { MHD Unstable, stabilized } \\
\text { by NBI + conducting shell }\end{array}$ \\
\hline Interchange, $n \geq 1$ & $\begin{array}{l}\text { MHD Unstable, stabilized by } \\
\text { compressional effects }\end{array}$ & Same as left column \\
\hline Rotational, $n=2$ & $\begin{array}{l}\text { MHD Unstable, stabilized by } \\
\text { quadrupole field and conducting shell }\end{array}$ & Same as left column \\
\hline
\end{tabular}

field, since $\rho_{\mathrm{i}} \sim \lambda_{\mathrm{i}}$ for $\beta \sim 1$ ). Several recent theoretical studies have considered the stabilizing effects of finite ion Larmor radius effects (ion FLR effects), the effects of the Hall term, and the effects of sheared ion flow on the $n=1$ tilt mode in prolate FRC configurations [8-13, 27, 41-47]. These studies have resulted in significant advances in the basic understanding of FRC stability properties. These advances include the following: (a) the role of ion FLR effects in affecting FRC stability properties has been clarified [8-13,42,47]; (b) the profile consistency of elongated FRCs has been determined, and a stability theory based on an expansion in the small parameter $1 / E$ (inverse elongation) has been developed [44,45]; (c) modern relaxation theory and the properties of two-fluid flowing equilibria have been studied [48-52]; (d) resonant particle effects have been demonstrated to be responsible for the instability drive in cases where FLR effects and the Hall term stabilize the MHD-unstable modes [10]; and (e) the nonlinear saturation of the $n=1$ tilt instability has been discovered in low- $S *$ configurations [11]. Progress has also been made in the area of collisionless pitch-angle scattering of plasma ions in the edge region of FRC plasmas [53], and studies of the confinement and current drive from neutral beam injection have moved forward $[54,55]$.

Recent theoretical research related to the SPIRIT concept has focused on investigating a variety of non-ideal MHD effects, including plasma flow and kinetic effects on FRC stability properties, particularly with respect to the $n=1$ tilt mode. A 3D nonlinear hybrid and MHD simulation code (HYM) has been developed [8]. The description of the numerical model and the results of extensive and systematic studies of stability properties for both prolate and oblate FRC configurations were presented in a series of [8-13]. Table 1 summarizes the most recent assessment of these stability properties in both prolate and oblate FRCs, based on the results from the HYM code and other studies. Many major, experimentally observed FRC stability properties have been explained with the help of these numerical simulations. In particular, the scaling of the linear growth rate of the $n=1$ tilt instability with the parameter $S * / E$ (Fig. 2) has been demonstrated for a

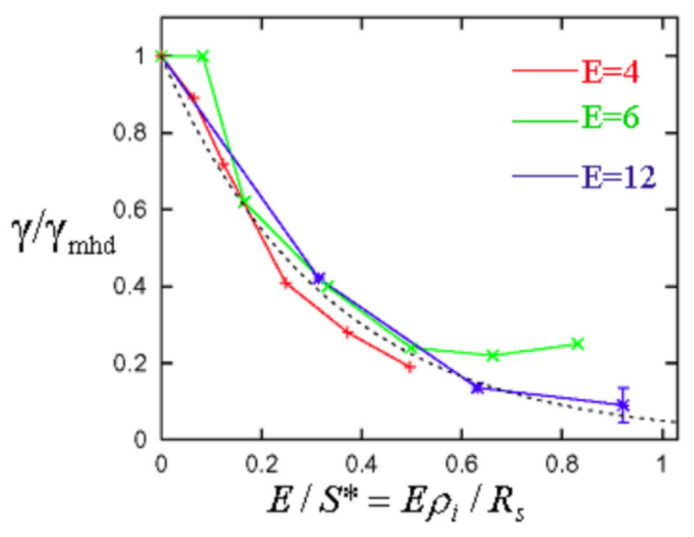

Fig. 2 Growth rates of the $n=1$ tilt instability for three elliptical FRC equilibria with $E=4,6$, and 12 [10].

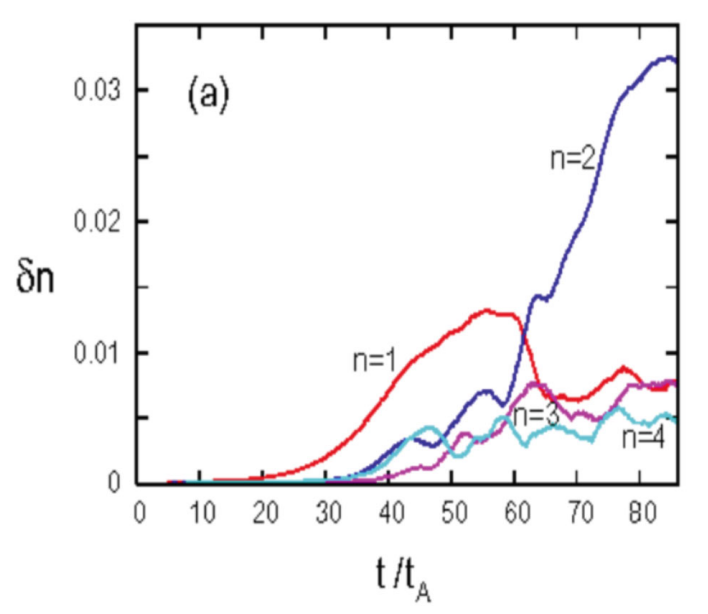

Fig. 3 Time evolution of the amplitudes of different $n$ modes in a prolate FRC with $S^{*}=20$ [11].

class of elongated elliptical FRCs [10], and the nonlinear saturation of the tilt mode (Fig. 3), and the growth of the $n=2$ rotational mode due to ion toroidal spin-up has been demonstrated [11]. For oblate FRCs, it has been shown that all $n=1$ modes (the tilt mode, the radial shift mode, 
and the interchange mode) can be stabilized in the MHD limit if a close-fitting conducting shell is present [9]. Interchange modes $(n>1)$ can be stabilized by pressure profile effects [9]. Furthermore, the low- $n(n>1)$ co-interchange modes have been shown to be the most dangerous modes for these configurations [9]. Finally, the effects of sheared flows have been studied in the MHD regime, and sheared flows are found to be stabilizing for large rotation rates (with Mach number $M>1$ ) [8].

The HYM code has also been used to study FRC formation by counter-helicity spheromak merging in support of the Swarthmore SSX-FRC experiment [12,22]. These simulations have successfully explained several puzzling experimental observations, including the incomplete reconnection of the toroidal fields, and the slower-than-MHD growth rate of the tilt mode [12]. A comparison between the HYM simulation results and the extensive experimental data from earlier FRC experiments (theta-pinch-formed prolate FRCs), as well as from the recent results from merging FRC experiments (TS-3 and the SSX-FRC), has demonstrated that the HYM code can reliably describe FRC plasmas.

Several of the key theoretical results relevant to the SPIRIT program are briefly summarized below.

Scaling of $n=1$ Tilt Mode Stability Properties: Figure 2 shows the results of recent hybrid simulations [10], performed for elliptical equilibria with uniformlyslow $z$-variation $[44,45]$ and a wide range of values of the elongation $E$. The growth rate of the internal tilt is plotted against the parameter $S * / E$, where the normalization is defined as $\gamma_{\mathrm{MHD}}=V_{\mathrm{A}} / Z_{\mathrm{s}}$, where $Z_{\mathrm{s}}$ is the separatrix half-length. The simulations demonstrate for this class of equilibria that the normalized tilt mode growth rate is a function only of the parameter $S * / E$. This supports the empirical stability scaling [40] with the parameter $S * / E$, which is based on experimental data, and suggests that this class of equilibrium profiles is realized in the experiments.

Nonlinear Evolution of Unstable $n=1$ and $n=2$ Modes: The nonlinear evolution of the unstable $n=1$ tilt mode and the $n=2$ rotational mode in the kinetic regime is illustrated in Fig. 3, which shows the time evolution of Fourier harmonics of the density perturbation. These nonlinear hybrid simulations demonstrate that the tilt instability can saturate nonlinearly at low values of $S^{*}$. The $n=2$ rotational mode is observed to grow during the nonlinear phase of the tilt instability due to ion spin-up in the toroidal direction. The ion toroidal spin-up is shown to be related to the resistive decay of the internal flux, and the resulting loss of weakly confined particles from the closed-field-line region $[11,12]$. Furthermore, the mechanism for nonlinear stabilization of the $n=1$ tilt mode has been discovered in the HYM simulations [11]. This research provides a clear explanation for the observation that the initial $n=1$ tilt motion in low- $S * / E$ experiments does not result in a total loss of confinement [56].
Oblate FRC Stability Properties with Conducting Shell: The global stability properties of oblate (small elongation, $E<1$ ) FRC configurations have been investigated numerically using both 3D MHD and hybrid simulations [9]. It is found that the $n=1$ tilt mode becomes an external mode when $E<1$, and that this mode can be effectively stabilized by a close-fitting conducting shell, even in the small-Larmor-radius (MHD) regime. Interchange mode stability properties are strongly profile dependent, and all $n \geq 1$ interchange modes can be stabilized for a class of pressure profiles with a separatrix beta larger than 0.035 . The simulation results [9] show that all $n=1$ modes can be stabilized in the MHD regime when passive stabilizers are utilized, but additional means of stabilizing the $n>1$ co-interchange modes are required.

Effects of Energetic Beam Ions: Thermal ion kinetic effects play a major role in stabilization of global MHD modes in low $-S^{*}$ prolate FRC experiments. Injection of energetic beam ions may provide an additional stabilizing mechanism in both prolate and oblate FRCs, provided that the beam ions carry a significant fraction of the total plasma current $[57,58]$. In addition, the injection of energetic beam ions is expected to contribute to plasma heating and sustainment of the FRC configuration. The lowfrequency stability properties of a hybrid system in which field reversal is created by both plasma currents, as in the FRC configuration, and by a low-density energetic component of large-orbit ions, have been studied previously by means of a generalized energy principle [59], and also by using 3D numerical simulations [58]. It has been shown for the low- $n$ co-interchange (kink) modes that the energetic ion beam contribution is stabilizing provided the condition $n|\Omega|>\omega_{\beta}$ is satisfied $[57,60]$, where $\Omega$ is the ion toroidal rotation frequency, $\omega_{\beta}$ is betatron frequency, and $\omega \ll \Omega$ is assumed. Numerical simulations of the tilt instability including the effects of the energetic beam ions have been performed [58]. In some of the cases considered, stabilization of the tilt instability was obtained for normalized beam ion density $n_{\mathrm{b}} / n_{\mathrm{e}}<2 \%$, and large beam ion velocities $V_{\mathrm{b}} \geq 10 V_{\mathrm{A}}$, where the Alfven speed $V_{\mathrm{A}}=B_{\mathrm{Z}} / \sqrt{\mu_{0} n m}$ is defined in term of the peak density and edge field strength. It was found that the beam particles tend to coalesce near the midplane, which reduces the efficiency of stabilization by energetic ions. Beam injection with large axial velocities was proposed for broadening the resulting profiles of the beam ions. A new numerical study of the effects of the energetic beam ions on global modes in the FRC has been performed recently using the HYM code[13]. Selfconsistent hybrid simulations including the full ion orbit effects in the realistic FRC geometry have shown that linear stability condition is more complex than that derived earlier $[59,60]$ in the bicycle-tire or infinite-length limits due to the possibility of secondary resonances of the general form: $n \Omega-\omega=l \omega_{\mathrm{Z}}+m \omega_{\mathrm{R}}$ (where $l$ and $m$ are integers; $\omega_{\mathrm{Z}}$ and $\omega_{\mathrm{R}}$ are axial and radial betatron frequencies). Linearized simulations have shown that these resonances (par- 
ticularly the one with $l=2, m=0$ for $\left.\omega_{\mathrm{Z}}<\omega_{\mathrm{R}}\right)$ can contribute significantly to stabilization or destabilization of the low- $n$ kink modes depending on the beam parameters and the mode polarization. More importantly, nonlinear hybrid simulations have demonstrated that the beam-driven instabilities are not dangerous, because they saturate nonlinearly at low amplitudes by changes in the beam ion distribution function [13]. Additional stabilizing effects are expected even after the beam ions are slowed down but remain hot due to the large ion-ion collision time, by effectively reducing the parameters $s$ through effects related to ion gyro-viscosity [61].

\subsection{Recent advances in FRC experiments}

Substantial progress has been made on all three of the important areas of FRC experimental research: (1) slow formation, (2) stability and confinement, and (3) current drive and sustainment. Below we briefly summarize the progress in each of these areas in the following sections.

Slow Formation of FRC Plasmas: Traditionally, FRCs are formed by the theta-pinch method (Ref. [2] and references therein), which requires high-voltage, pulsed operation. This method suffers from difficulties in applicability to reactor-relevant devices. Alternate methods are being pursued to form large-flux FRCs in a more controlled manner. One method is based on the Rotating Magnetic Field (RMF), which was first explored by Jones [62], and has been successfully applied [34] in the TCS (Translation, Confinement, and Sustainment) device at the University of Washington. Field reversals up to $200 \mathrm{G}$ have been obtained by applying an RMF to pre-ionized plasma in a uniform axial field of $150 \mathrm{G}$. Although the temperature was relatively low $(40 \mathrm{eV})$, the slow formation of FRC plasmas was clearly demonstrated. An alternative method is based on the merging of counter-helicity spheromaks, a method developed at TS-3/4 [14-20], as shown in Fig. 4. The annihilation of oppositely-directed toroidal field during merging is converted to a large increase in ion temperature up to $200 \mathrm{eV}$ [16] with a large poloidal flux of up to a few $\mathrm{mWb}$ in a relatively small facility [15]. More recently, this technique was also successfully applied [22] to form FRC plasmas in the SSX device and in the MRX device [23,24].

Stability and Confinement of FRC Plasmas: The stability and confinement properties of FRC plasmas have been extensively studied in FRC experimental research. Recent results with neutral beam injection into FRC plasmas formed by the theta-pinch method at FIX (FRC Injection Experiment) indicated that the global $n=1$ mode movement is slowed by neutral beam injection [63]. Due to the limited poloidal flux in FIX, the beam was injected with a large angle along the axial direction. The stabilizing effects of ion rings confined by mirror fields at each end have been suggested. As an accompanying effect, the plasma confinement was claimed to improve $[64,65]$. The characteristic decay time of the FRC volume was observed to increase from $95 \mu$ s to $230 \mu$ s with neutral beam injection. Although the detailed information on the beam ions, such as the slowing-down process and charge exchange losses due to background neutrals, was not available, these global results are encouraging. Stability and confinement effects due to the RMF have also been explored [33] in FRC experiments, which can be attributed to two-fluid effects produced by rotational or ponderomotive forces. More recently, the stabilizing effects of the RMF on the $n=2$ in-

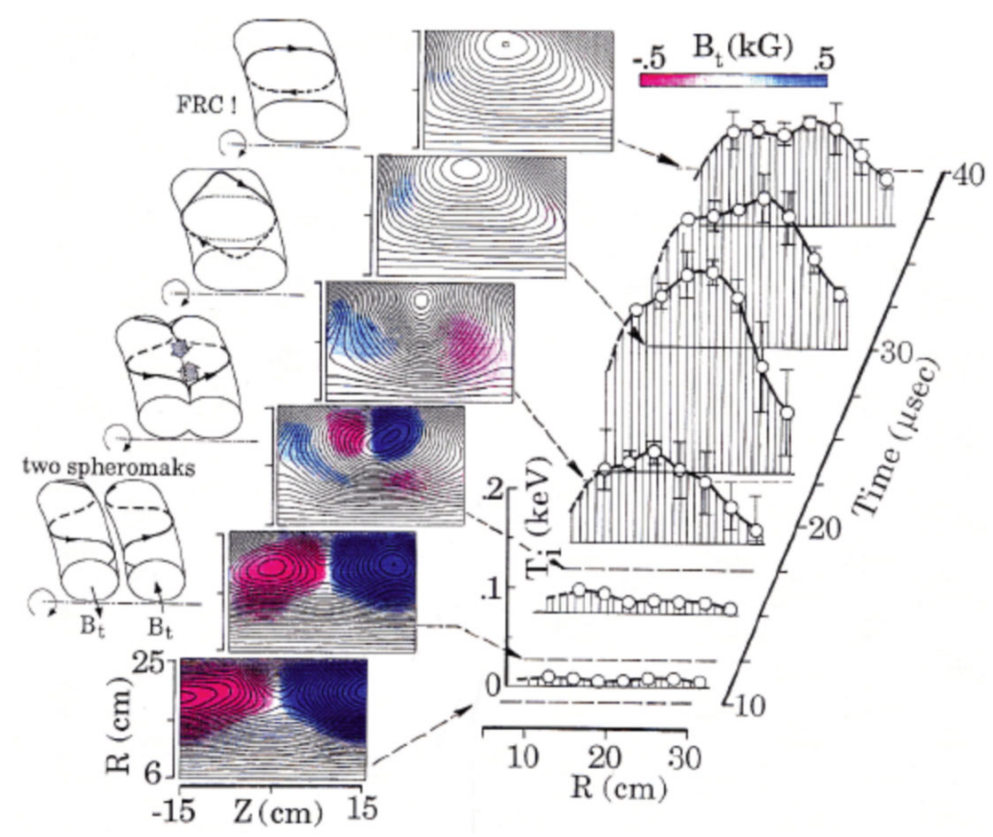

Fig. 4 Time evolution of the flux contours, toroidal field (red for negative values and blue for positive values), and ion temperature during the formation of an FRC plasma by merging counter-helicity spheromaks. Figure courtesy of Y. Ono, U. of Tokyo. See Ref. [16] for additional details. 
terchange mode or the rotational mode have been reported for the first time [35].

Current Drive and Sustainment of FRC Plasmas: Current drive and sustainment beyond a resistive decay time is another important requirement for the FRC to be reactor-relevant. The primary solution candidates are RMF [33-35,66-68] and neutral beam injection [54,55,69, 70]. In both the TCS and STX (Star Thrust Experiment) devices, long-time current drive by RMF was successfully demonstrated $[36,71]$. Field reversal was maintained as long as the RMF was supplied. The plasma perpendicular resistivity, which determines the decay rate of the magnetic flux, was shown to be enhanced over its classical values by a factor of 10-30 depending on the RMF amplitude [72]. More recently, it has been shown [73] that when the RMF frequency is high, the electrons are less synchronous with the RMF, and the RMF only penetrates into the outer edge region. It was suggested that a reconnection process transfers the current inwards toward the magnetic null region. The perpendicular plasma resistivity may have a profile that peaks at the edge, possibly due to the large RMF amplitude there. In contrast to RMF, experiments using neutral beam tangential injection to sustain FRC plasmas have never been performed despite early proposals $[69,70]$. Current sustainment by fusion charged particles has also been studied $[74,75]$.

Current drive with a transformer at the major axis of an FRC was experimentally demonstrated in the TS-3 experiments, in which an FRC configuration was sustained for a substantially long time [15]. While the detailed relaxation mechanisms were not identified, we expect that the following basic mechanism was at work in this experiment.

When an ohmic transformer is turned on, it generates a one-turn voltage in the azimuthal direction by a flux swing, thus creating an electric field in the azimuthal direction. This electric field induces a current as well as an inward plasma motion at the plasma surface through the relationship

$$
E_{\theta}+V_{\mathrm{r}} \times B_{\mathrm{p}}=\eta_{\perp} j_{\theta},
$$

where $\eta_{\perp}$ is the perpendicular resistivity, and $\eta_{\perp}=2 \eta_{\|}$. With an external driving field, $E_{\theta}$ would compress the plasma by the $V_{\mathrm{r}} \times B_{\mathrm{p}}$ force, thus inducing a stronger pressure gradient and increasing the (diamagnetic) plasma current. Under quasi-steady-state conditions, a continuous inflow of poloidal flux should be balanced by the dissipative loss of current inside the plasma. This mechanism is similar to ohmic current maintenance in tokamaks except for the fact the current flows perpendicular to magnetic field in FRC plasmas.

Recent experiments in TS-4 have added to our knowledge of Ohmic-sustainment of an FRC [21]. These experiments showed the sustainment of an FRC configuration for $>300 \mu \mathrm{s}$, without the growth of destructive instability utilizing argon as the working gas. The termination of the configuration was due to a loss of equilibrium field due to the stray return flux from the transformer. Furthermore, these experiments have demonstrated an increase in the effective perpendicular resistivity during the Ohmic ramp, allowing magnetic energy to be converted to thermal energy and maintaining the balance between magnetic and thermal energy.

\section{SPIRIT Scoping Studies}

In order to assess the feasibility of the SPIRIT concept, a series of scoping studies on the relevant topics, both theoretical and experimental, have been carried out. A summary of the obtained results is described in this section. In particular, numerical studies have revealed an operating regime in which the FRC plasmas are stable to all global MHD modes by utilizing stabilizing effects from both the beam ions and a conducting shell for oblate FRCs. Experimentally, a series of preliminary studies have been carried out that successfully demonstrate the slow formation of FRC plasmas in the present MRX facility.

\subsection{Counter-helicity spheromak merging simulations}

Two-dimensional MHD simulations of counterhelicity spheromak merging have been performed for MRX geometry using the HYM code. The initial conditions were assumed to correspond to two spheromaks with $a \approx 15 \mathrm{~cm}$ and separation $\sim 2 a$, with the following initial plasma parameters: $n_{\mathrm{e}}=10^{14} \mathrm{~cm}^{-3}$, central field $B_{0}=1.3 \mathrm{kG}$, and $T_{\mathrm{i}}+T_{\mathrm{e}}=45 \mathrm{eV}$. Uniform resistivity and viscosity were assumed in the simulations, with Lindquist number $S=2000$ and Reynolds number $R_{\mathrm{e}}=1000$. The merging continued for about $t=15-20 t_{\mathrm{A}}$, and an FRC configuration was formed with elongation of order of one ( $E=1-1.2$ ), separatrix radius $R_{\mathrm{s}}=39 \mathrm{~cm}$, and approximately flat current profile (the external poloidal field was $B \approx 1 \mathrm{kG}$ ). Significant toroidal flows were generated during the reconnection phase, up to $V=0.9 V_{\mathrm{A}}$, but these were reduced to $V<0.05 V_{\mathrm{A}}$ after the FRC formation was completed. Most of the reconnected magnetic field energy was converted into plasma thermal energy, with a resulting total temperature of the FRC plasma $T_{\mathrm{i}}+T_{\mathrm{e}}=300 \mathrm{eV}$. Results from these simulations are shown in Fig. 5, where the contours of poloidal flux are plotted at three different times during the merging. Another set of numerical simulations has demonstrated that the parameters and shape of the final FRC configuration can be controlled by varying the initial spheromak parameters, the field of the separation coils, and the mirror ratio of the external coils.

\subsection{Numerical study of FRC stability prop- erties}

Stability properties of an FRC configuration similar to that in Fig. 5 have been studied using the hybrid version of the 3D simulation code HYM. A full-orbit kinetic de- 

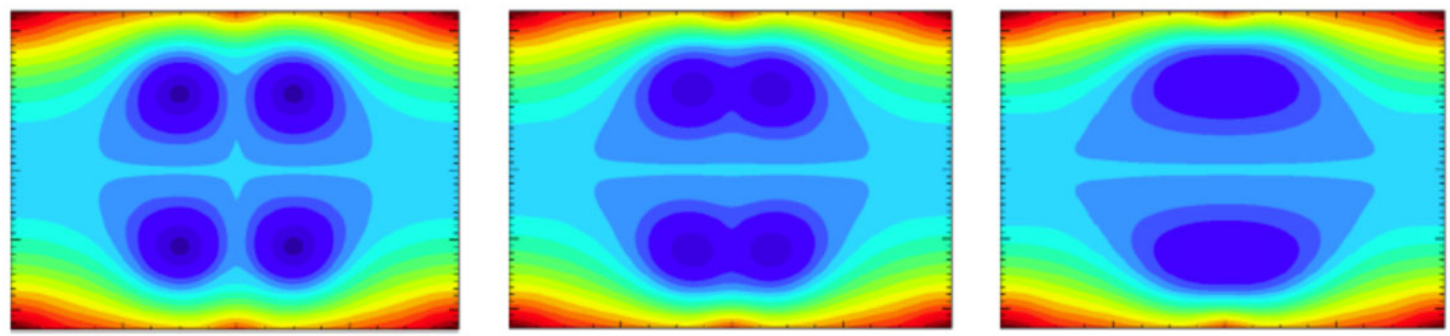

Fig. 5 Contour plots of poloidal flux from 2D MHD simulations of counter-helicity spheromak merging. An FRC plasma with $E=1.2$ is formed. The three frames are at $5 \tau_{\mathrm{A}}, 10 \tau_{\mathrm{A}}$, and $20 \tau_{\mathrm{A}}$.

scription is used for both the thermal ions and the beam ions, and the electrons are treated as a cold fluid. Linear and nonlinear stability properties of the MHD modes with toroidal mode numbers $n=1-4$ have been investigated. For parameters of typical oblate FRCs in MRX, the thermal ion kinetic parameter is $S^{*} \approx 18$, and the elongation is $0.5<E<2$, so that the stability parameter $S^{*} / E \sim 10-20$ is well above the empirical stability boundary at $S * / E=3$ 4. The growth rates of the $n=1$ tilt mode and the $n>1$ co-interchange (kink) modes for this case are $\gamma \sim \gamma_{0}$ (where $\gamma_{0}=V_{\mathrm{A}} / Z_{\mathrm{s}}$ is the MHD growth rate). However, for $E \sim 1$, the $n=1$ tilt mode becomes an external mode, and its growth rate is reduced by an order-of-magnitude when a close-fitting conducting shell is used for stabilization (Fig. 6 (top)). A similar reduction of the tilt growth rates has been found in simulations for larger elongations $1<E<2$. The growth rates of the $n>1$ modes are also reduced due to conducting-shell effects. Conducting-shell stabilization for these parameters is stronger than was previously found for MHD plasmas [9] due to the change in the linear mode structure caused by thermal ion kinetic effects. With conducting-shell stabilization, the $n=2$ axially polarized co-interchange mode becomes the most unstable mode (Fig. 6 (bottom)). The growth rate of the $n=3$ mode is also reduced and is smaller than that of the $n=2$ mode. The higher- $n$ modes, $n>3$, are stabilized by the combination of the thermal ion finite Larmour radius and the conducting shell. The localization of the unstable low- $n$ modes near the magnetic null suggests that neutral beam injection may be effective in stabilizing these residual instabilities.

Stability properties of these configurations have also been studied including the effects of the energetic beam ions [13]. The exponential rigid-rotor distribution was assumed for the energetic ions, i.e., $f_{\mathrm{b}}(\varepsilon, p)=A \exp [-(\varepsilon-$ $\left.\Omega_{0} p\right) / T_{\mathrm{b}}$, where $\varepsilon$ is the ion energy, $p$ is the toroidal canonical angular momentum, and $T_{\mathrm{b}}$ is the (constant) beam ion temperature. This distribution function corresponds to a local shifted Maxwellian distribution function with constant angular rotation frequency $\Omega_{0}$. For MRX parameters and a beam ion energy of $20-25 \mathrm{keV}$, the beam ion injection velocity is large compared to the Alfven velocity, $V_{0} \approx 10 V_{\mathrm{A}}$, and the peak beam ion density $\left(n_{\mathrm{b}}\right)$ is $1-3 \%$ of
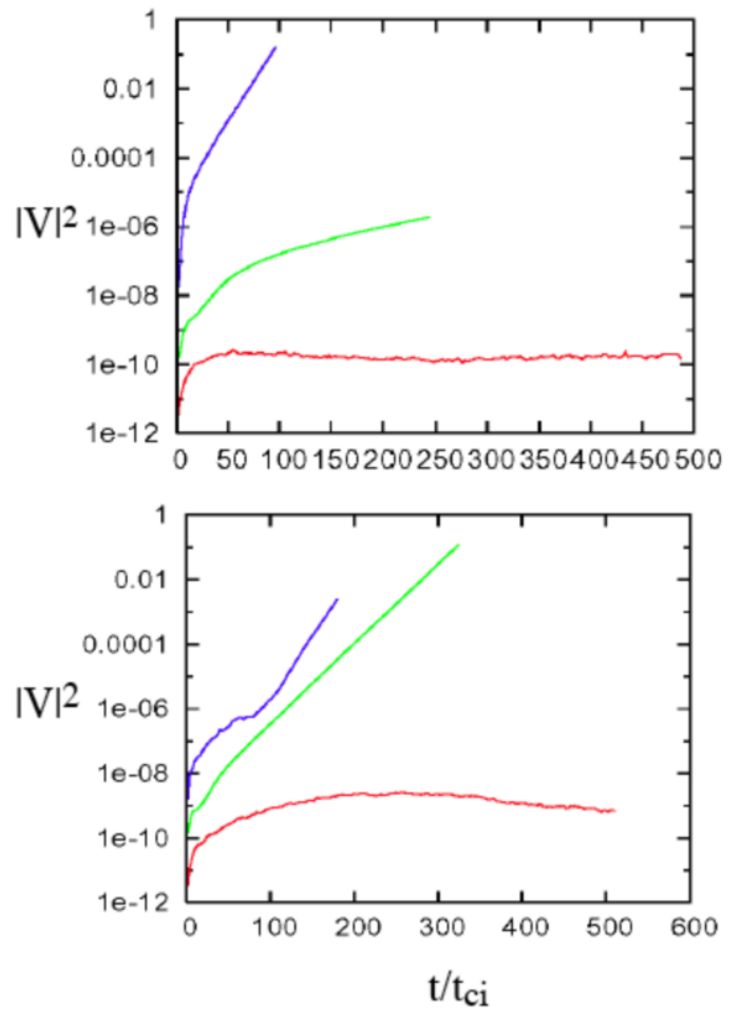

Fig. 6 Time evolution of the $n=1$ (top) and $n=2$ (bottom) components of the perturbed kinetic energy from 3D hybrid simulations without additional stabilization (blue), and including the effects of a conducting shell (green), and beam stabilization (red).

the background density. Self-consistent hybrid simulations show that the neutral-beam-injected current tends to localize between the magnetic null and the separatrix at the FRC midplane, and even though the fraction of the total current carried by the neutral-beam-injected ions is small compared to the bulk plasma current, the local neutral-beaminjected current density $J_{\mathrm{b}}$ is comparable to the thermal plasma current density for $n_{\mathrm{b}} \geq 2 \%$ of the background density. Since the internal MHD modes are also localized near the magnetic null, the energetic beam ions have a strong stabilizing effect on these modes. 
Linearized hybrid simulations have been performed for various beam ion parameters: toroidal velocity of $V_{\mathrm{b}} \approx$ 4- $6 V_{\mathrm{A}}$, and normalized peak density of $n_{\mathrm{b}}=1.5-3 \%$. It is found that the energetic beam ion stabilization is more effective for broader beams (larger beam ion temperature), and for FRCs with elongation $E \sim 1$. When $E \sim 1$, the neutral beam stabilization is very effective for the $n=1$ and $n=2$ modes, which are stabilized completely for the following beam parameters: $V_{\mathrm{b}}=6.1 V_{\mathrm{A}}, T_{\mathrm{b}} /\left(m_{\mathrm{i}} V_{\mathrm{A}}^{2} / 2\right)=$ 10 , and $n_{\mathrm{b}}=3 \%$. The energetic beam ions also reduce the growth rate of the $n=3$ co-interchange mode, but this mode remains weakly unstable for these parameters.

A new set of 3D nonlinear hybrid simulations have been performed in order to study the nonlinear evolution of the FRC in the presence of the conducting shell and the energetic beam ions [13]. These simulations show that the residual $n=3$ instability saturates nonlinearly at low amplitude (Fig. 7), and therefore it is not a dangerous mode. Simulation runs which model the "sustained" FRC configuration (i.e., without decay of the equilibrium current) show that after the $n=3$ mode saturates, the resulting configuration remains stable with respect to all global MHD modes, as long as the FRC current is sustained (Fig. 7). In contrast, when the configuration was allowed to decay resistively, the slow reduction in the separatrix radius resulted in the reduction of the conducting shell effects, and eventual destabilization of the $n=1$ tilt mode occurs at $t=800 t_{\mathrm{ci}}$ (simulations not shown). Therefore, the ability to sustain the configuration (by NBI and current transformer) is essential, and it should allow the experimental achievement of the stable operating regime.

These results are summarized in Table 2, where the impact of each stabilizing mechanism is discussed. The cell in the lower right describes the stabilizing methods that lead to an FRC that is stable to all global modes. Note that this is a new stability regime (i.e., a combination of small elongation, conducting shell, and beam ion effects), which has never been experimentally studied, and can potentially lead to new directions in FRC research.

The stabilizing effects of the neutral-beam-induced bulk plasma rotation have not been included in present numerical study, but these will likely contribute to the stabilization of the low- $n$ MHD modes. Future theoretical studies are anticipated in order to optimize the beam and bulk plasma parameters and profiles with respect to stabilization of the low- $n$ MHD modes.

\subsection{Experimental scoping study of FRC for- mation in MRX}

In addition to the computational studies of spheromak merging and FRC stability, we have conducted preliminary

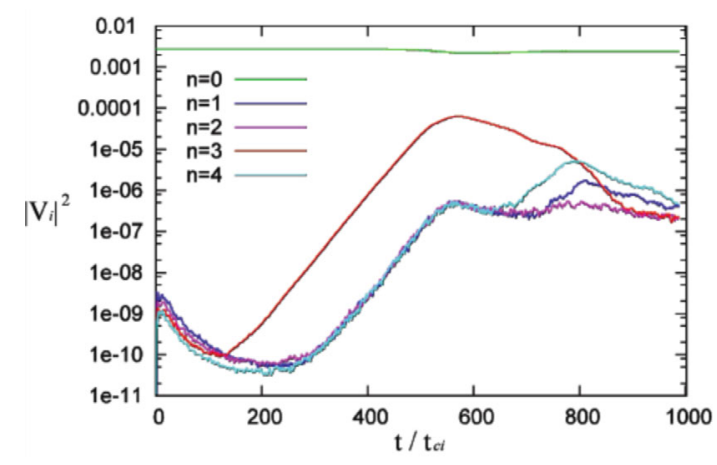

Fig. 7 Time evolution of the $n=0-4$ components of the ion kinetic energy from 3D nonlinear hybrid simulations including the effects of a conducting shell and beam stabilization. The $n=3$ mode is the linearly unstable mode, which saturates nonlinearly at $t=550 t_{\mathrm{ci}}$ (apparent growth of other modes at $t=200-500$ is due to weak numerical coupling to the $n=3$ mode). The Alfven time is $t_{\mathrm{A}}=20 t_{\mathrm{ci}}$.

Table 2 Effects of different stabilizing mechanisms on FRC stability properties, based on experimental and computational results. The configuration in the bottom right, corresponding to the SPIRIT concept, is predicted in simulation to be stable to all global modes. Testing this prediction represents a unique opportunity for FRC research.

\begin{tabular}{|c|l|l|}
\hline $\begin{array}{c}\text { Stabilizing } \\
\text { mechanisms }\end{array}$ & \multicolumn{1}{|c|}{ Prolate FRC $(E>1)$} & \multicolumn{1}{|c|}{ Oblate FRC $(E \sim 1)$} \\
\hline $\begin{array}{c}\text { Toroidal Rotation, } \\
\begin{array}{c}\text { Nonlinear Effects, } \\
\text { and Multipole } \\
\text { Stabilization }\end{array}\end{array}$ & $\begin{array}{l}\text { Unstable (the } n=1 \text { tilt and higher- } n \\
\text { MHD modes) except for long } \\
\text { kinetic configurations with } S^{*}<20 \\
\text { and } E \geq 5 \text { (theta-pinch-formed FRCs) }\end{array}$ & $\begin{array}{l}\text { Unstable (the } n=1 \text { tilt and higher- } \\
n \text { MHD modes) }\end{array}$ \\
\hline Conducting Shell & Unstable (No effect on stability) & $\begin{array}{l}\text { Unstable (Axially polarized } n>1 \\
\text { kink modes) }\end{array}$ \\
\hline NBI & Unstable (the $n=1$ tilt mode) & $\begin{array}{l}\text { Unstable (Some of the } n \geq 1 \text { modes } \\
\text { are stabilized depending on the } \\
\text { mode polarization and the NBI } \\
\text { parameters.) }\end{array}$ \\
\hline $\begin{array}{c}\text { NBI, Conducting } \\
\text { Shell, and Multipole } \\
\text { Stabilization }\end{array}$ & Unstable (the $n=1$ tilt mode) & $\begin{array}{l}\text { Stable (with respect to all global } \\
\text { modes) }\end{array}$ \\
\hline
\end{tabular}



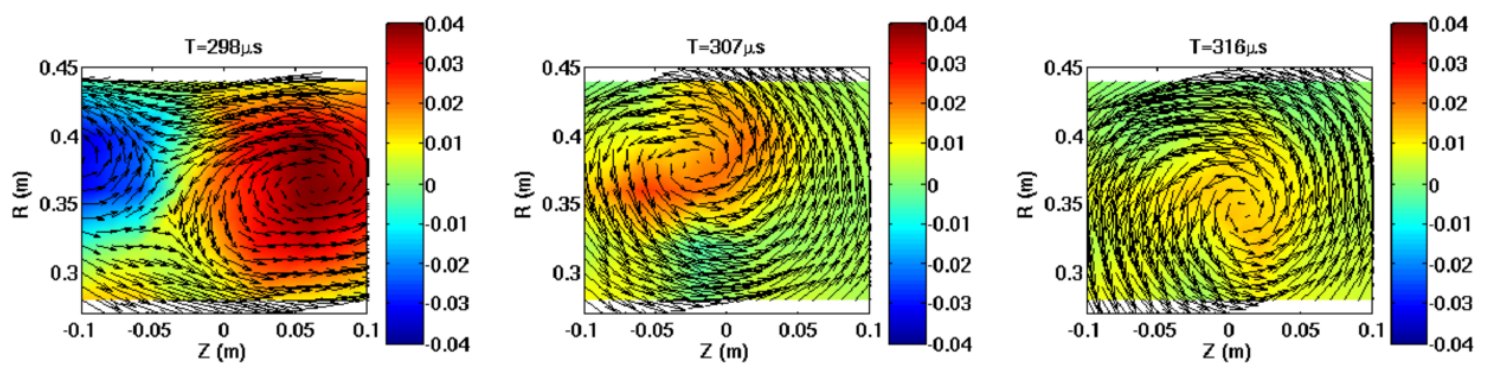

Fig. 8 Snap-shots of the magnetic field at three times during the merging process in MRX. The poloidal magnetic field is represented by arrows, and the toroidal magnetic field by colors. The toroidal field scale on the right of each figure is in units of Tesla.

merging experiments in the MRX device. These studies have demonstrated the feasibility of plasma merging for FRC formation in the MRX device.

We are able to diagnose the dynamics of the plasma merging using a two-dimensional array of magnetic pickup coils centered on the merging region. This array measures all three components of the magnetic field at each location, providing detailed information on the magnetic structure. In Fig. 8, the merging sequence for a helium discharge is illustrated at three times. The flux cores are located at $Z= \pm 0.4 \mathrm{~m}$, outside the range of the measurement. At $t=298 \mu \mathrm{s}$, the two spheromaks have pinched off from the flux cores and are approaching each other. At $t=307 \mu \mathrm{s}$, the poloidal fields of the two spheromaks have reconnected, but the toroidal fields are still present and finite. At $t=316 \mu$ s, the toroidal fields have settled to a small final value (maximum $\left(B_{\mathrm{T}} / B_{\mathrm{p}}\right)<20 \%$ ), and the FRC plasmas is formed. The FRC plasma then decays away, possibly due to an instability.

This merging process can lead to significant ion heating, in agreement with previous results [16]. The ion temperature evolution is shown in Fig. 9, for helium discharges. The shaping field coils are used to compress the spheromaks before merging in this case, leading to large values of the reconnecting fields. The ion temperature is measured using a spectroscopic probe [76] located at $(R, Z)=(0.375,0)$ meters. The ion temperature before merging is about $10 \mathrm{eV}$. During the merging process, the temperature increases to $30 \mathrm{eV}$, significantly exceeding the electron temperature (measured by a triple Langmuir probe). This reconnection-driven ion heating may provide an important pathway to establishing kinetic FRCs in the MRX device.

These initial experimental results provide considerable confidence that the SPIRIT concept can indeed be studied in an MRX-like facility. It is anticipated that future experiments utilizing passive conducting structures for stability and Ohmic sustainment should make an attractive target plasma for neutral beam injection experiments.

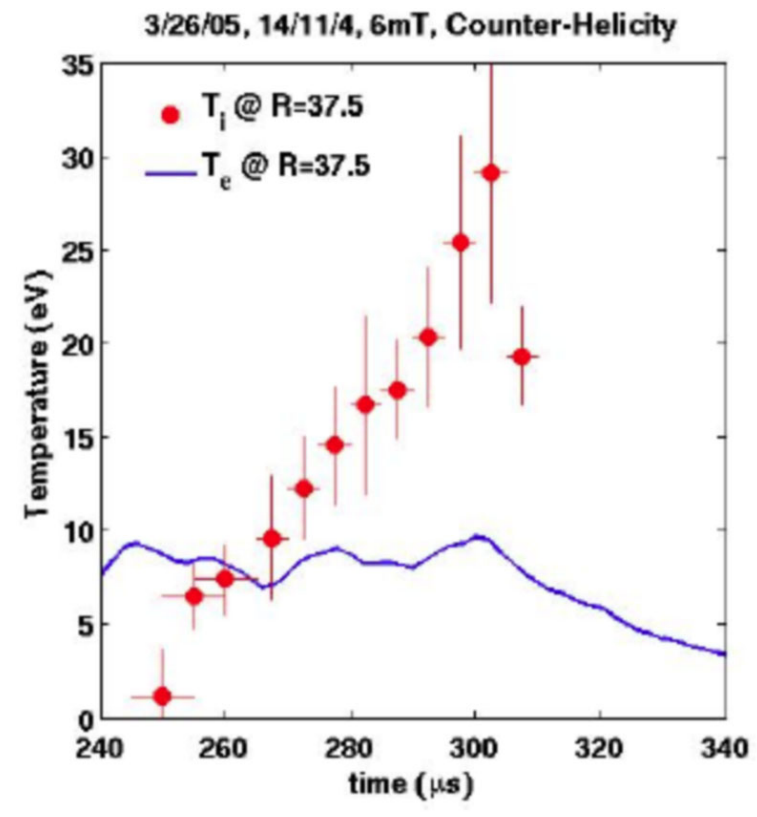

Fig. 9 Ion temperature rise during merging of Helium-plasma spheromaks. The ion temperature vastly exceeds the electron temperature, illustrating the heating inherent in merging plasmas.

\section{Research Plan for SPIRIT Studies in the MRX Device}

In this section we describe a series of experiments on the MRX device to investigate the MHD stability properties of FRCs by maintaining such plasmas for times much longer than the energy confinement time. If carried out, these experiments would test many of the important ideas in the SPIRIT concept. The major objectives of these experiments are to:

(1) Form large-flux FRCs by the merging of two spheromak plasmas with opposite helicities that are created by the flux-core induction scheme, and increase the total flux of the FRC (up to $30 \mathrm{mWb}$ ) with the aid of a current transformer;

(2) Experimentally assess the global stability properties of FRCs by varying the plasma shape (especially the 
elongation $E$ ), the passive stabilizers, and the kinetic parameter $s$ (number of gyro-radii between magnetic axis and the separatrix radii) with the use of neutral beam injection, and compare the experimental data with state-of-theart numerical simulation results; and

(3) Sustain the FRC plasmas for significantly longer (>1 msec) than the energy confinement time, with the aid of a current transformer and neutral beam injection.

The following sections provide details regarding three important aspects of this experimental program: FRC formation, sustainment with current transformers, and the confinement of high-energy ions from the neutral beam. The experiments in the MRX device are used to describe a specific and testable manifestation of the SPIRIT concept; different aspects of the SPIRIT concept could be tried in various oblate FRC experiments, though the details would be different.

\subsection{FRC formation and conducting shell stabilization}

The FRC plasmas are formed by merging two spheromak plasmas with opposite helicities. The spheromak plasmas are generated by induction at each end of the vessel by appropriately programming the currents inside the flux cores. The original spheromak formation scheme used for $\mathrm{S}-1$ was based on a single flux core [77]. The FRC formation technique employed in MRX is based on two flux cores, a new scheme developed in MRX [23, 24] and TS3 [14-21]. Plasma merging is also being studied on the Swarthmore SSX device using spheromak guns [22]. By pulsing the TF and PF currents in the flux cores on both sides of the formation region, two plasmas are created by induction, as shown in Fig. 10 (a). When a current channel grows near the flux cores, spheromak plasmas are formed (Fig. 10(b)). A pair of additional poloidal coils (shaping field coils) located near the midplane prevents the two formation regions from interfering with each other. The size of the two spheromak plasmas is expected to be $R \leq 30 \mathrm{~cm}$ and $a \leq 25 \mathrm{~cm}$, corresponding to 20-30 ion gyroradii. A Lundquist number $S>2,000$ is expected for the initial spheromak plasmas based on the experience in S-1. The two spheromak plasmas, which carry identical toroidal currents with opposite toroidal field, are then allowed to merge along a common axis due to their attractive force (Fig. 10 (c) and (d)). Currents in the separation coils and flux cores exert forces on the plasmas to control the merging rates and alter the large-scale equilibrium configuration. Our preliminary experiments have utilized merging to produced FRCs with flux in the $3-10 \mathrm{mWb}$ range, and anticipate that the when operated at full parameters, the FRCs so produced in MRX could have a flux of $\sim 20 \mathrm{mWb}$. This flux would then decay unless sustained by NBI or the current transformers.

The separatrix radius of the formed FRC plasma is expected to be up to $45 \mathrm{~cm}\left(R_{\mathrm{S}}\right)$, substantially larger than that previously obtained. The timescale for spheromak formation can be varied from 30 to $100 \mu \mathrm{sec}$ by changing the effective inductance and capacitance in the circuit. Based on preliminary experimental results obtained at the University of Tokyo ( $70 \%$ of the toroidal field energy was converted to ion kinetic energy), it is expected that a very high pressure plasma with $T_{\mathrm{i}}>0.5 \mathrm{keV}\left(T_{\mathrm{e}}>50 \mathrm{eV}\right)$ and $n_{\mathrm{e}}>10^{14} \mathrm{~cm}^{-3}$ can be obtained immediately after plasma merging.

Conducting shells are an important component of the (a)



(c)



(b)

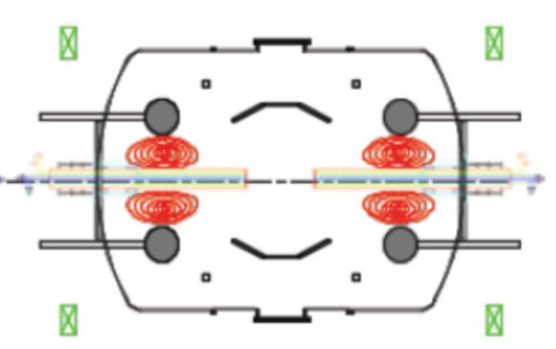

(d)



Fig. 10 Conceptual illustration of formation process of FRC plasmas in MRX with current transformers. 
SPIRIT concept: they provide stability to $n=1$ modes (and higher- $n$ modes as well) during both the merging and FRC equilibrium phases. The present design calls or the shells to be made from four different copper segments, so that equilibrium-distorting toroidal currents cannot flow between the shells. The shells will be movable, in order to modify the radius of the outer plasma boundary.

\subsection{Current drive transformers}

Current drive transformers are used to sustain and amplify the flux of the FRC. Two movable solenoids, insertible from either end of the device, are utilized to induce toroidal current in the plasma, and provide up to $50 \mathrm{mWb}$ of flux swing. A water-cooled central tube provided in the center of the assembly is used to conduct the heat from the conductor coils. These transformers are enclosed in thin-wall Inconel vacuum jackets, to allow rapid ramp-up of the plasma current. A copper conductor with an axial cut is placed under the Inconel liner. This conductor provides stabilization to $n=1$ modes, while the axial cut allows the Ohmic flux to escape. Variable tap connections in the circuit allow different combinations of maximum voltage and pulse length. A decoupling transformer is provided to avoid the coupling of the transformer current to the poloidal flux (PF) circuit for the flux cores. Note that current amplification by a transformer has been successfully applied to spheromaks formed by a flux core [78], and to FRCs formed by spheromak merging (see Sec. 2.2) [15].

This transformer system can be operated in three different fashions. When the transformers are fully inserted, they appear to the plasma as a single long solenoid, similar to that in Ref. [15]. The system in this case is operated in "single-swing" mode, where the solenoid current is ramped from zero in order to drive plasma current. Extra coils may be necessary to cancel some stray field from the transformer (which opposed the external field), as was done in the case of the transformer driven spheromak [77] and the TS-4 experiments [21].

In the second, more unique mode of operation, the transformers are retracted towards the flux-cores, leaving a large open volume in the center of the machine. The transformers are "loaded" with current before the spheromak formation phase, after which the solenoid current is ramped down, enhancing the spheromak current, In this "double-swing" mode, spheromaks with very large currents can be formed and merged, leading to an FRC with very large flux. This scheme is illustrated in the merging illustrated in Fig. 10. Note that the leakage flux from the transformer is not expected to interfere substantially with the spheromak formation phase, due to the large PF fields of the flux-cores, and that the transformers do not contribute any field during the FRC equilibrium phase.

The transformer system should provide sufficient flux and power to sustain the FRCs formed in MRX. For the nominal operating point of $T_{\mathrm{e}}=50 \mathrm{~V}$ (see Table 3), the collisional perpendicular resisitivity is given by $\eta_{\perp} \approx$ $4 \mu \Omega \cdot \mathrm{m}$. Based on the present experience on MRX and the results from many theta-pinch formed FRCs [2], we anticipate that the actual resistivity, augmented by some anomalous processes, will be $\sim 10$ greater than the collisional result. This assumption yields an anticipated resistivity of $\eta_{\perp} \approx 40 \mu \Omega \cdot \mathrm{m}$. For the current density of $500 \mathrm{kA} / \mathrm{m}^{2}$ anticipated at a current of $\sim 100 \mathrm{kA}$, the resulting electric field is $E=20 \mathrm{~V} / \mathrm{m}$, corresponding to a single-turn of $\sim 10 \mathrm{~V}$. This voltage allows the plasma to be sustained for $\sim 5 \mathrm{msec}$ for the anticipated $50 \mathrm{mWb}$ total transformer flux in the fully inserted transformer configuration. This time is more than sufficient for the goals of our experiment, and leaves comfortable headroom for even larger values of the resistivity anomaly.

For the reference scenario described above, the Ohmic power input to the plasma is in the range of 1-2 MW. For $T_{\mathrm{e}}=T_{\mathrm{i}}=50 \mathrm{eV}$, and $n=1 \times 10^{14} \mathrm{~cm}^{-3}$ and a plasma volume of $0.2 \mathrm{~m}^{3}$ (a reference scenario for an FRC sustained by the current transformer alone), this implies a stored energy of $200 \mathrm{~J}$, leading to a required energy confinement time of $\sim 0.1 \mathrm{msec}$. This is shorter than the expected value of the energy confinement time based on the scalings from theta-pinch formed FRCs [79]. Hence, it appears that the current transformer system is consistent with the input power and configuration lifetime requirements.

Table 3 Parameters of typical target FRC plasmas for neutral beam injection.

\begin{tabular}{|l|l|l|}
\hline Parameters & Target plasma (I) & Target plasma (II) \\
\hline Plasma current & $120 \mathrm{kA}$ & $180 \mathrm{kA}$ \\
\hline$B_{\max }$ & $1.0 \mathrm{kG}$ & $1.5 \mathrm{kG}$ \\
\hline Peak density & $1 \times 10^{14} \mathrm{~cm}^{-3}$ & $1 \times 10^{14} \mathrm{~cm}^{-3}$ \\
\hline$T_{\mathrm{e}}$ & $50 \mathrm{eV}$ & $50 \mathrm{eV}$ \\
\hline$T_{\mathrm{i}}$ & $300 \mathrm{eV}$ & $600 \mathrm{eV}$ \\
\hline$R_{\mathrm{p}}$ & $30 \mathrm{~cm}$ & $30 \mathrm{~cm}$ \\
\hline$R_{\mathrm{s}}$ & $40 \mathrm{~cm}$ & $40 \mathrm{~cm}$ \\
\hline$S^{*}$ & 18 & 18 \\
\hline Trapped flux & $15 \mathrm{mWb}$ & $22 \mathrm{mWb}$ \\
\hline
\end{tabular}




\subsection{Neutral beam injection into FRCs in MRX}

A major goal of the SPIRIT research program is to scientifically test the idea of steady-state sustainment and kinetic stabilization of FRC plasmas by neutral beam injection. We plan to inject a $20-30 \mathrm{keV}$ neutral beam with a 'current' of $\sim 60 \mathrm{~A}$ into the FRC plasma in MRX, in order to sustain the configuration for at least a millisecond. It is expected that this neutral beam injection would induce the plasma to spin with a high velocity of the order of $V_{\mathrm{A}}$, which would in turn help stabilize the global MHD modes (see Sec. 2.1). (The beam ion speed is about $10 V_{\mathrm{A}}$.) Neutral beam injection should also decrease the value of the kinetic parameter $s$. Tangential neutral beam injection can drive a toroidal current by three possible mechanisms: 1) direct ion ring current flow with unbalanced electron flow, although this effect may be limited due to the low electron temperature in the small MRX manifestation of SPIRIT; 2) the 2D dynamo effects due to the electron temperature gradient caused by electron heating near the axis [80] and 3) self-organization (dynamo) processes involving 3D non-axisymmetric modes. If we can identify the fundamental mechanism for current drive by neutral beam injection, it would constitute a major advance in plasma physics. Note that the current transformer system is present to sustain the bulk plasma flux in these MRX experiments, regardless of the ultimate efficiency of NB current drive.

A scoping study of neutral beam injection experiments has been carried out for the following basic parameters of the target FRC plasma and neutral beam injection system, with the energy balance described below.

(1) Target plasmas for the neutral beam injection calculations have peak density, $n_{\mathrm{e}}(0) \sim 1 \times 10^{14} \mathrm{~cm}^{-3}$, $T_{\mathrm{e}}(0) \sim 50 \mathrm{eV}, T_{\mathrm{i}}(0) \sim 0.5 \mathrm{keV}$. We have performed orbit simulations for plasmas with two different magnetic field strengths: Case I with $I_{\mathrm{p}}=120 \mathrm{kA}$, and $B_{\text {edge }}=1.0 \mathrm{kG}$, and Case II with $I_{\mathrm{p}}=180 \mathrm{kA}$, and $B_{\text {edge }}=1.5 \mathrm{kG}$. The total stored energy is $\sim 1.7 \mathrm{~kJ}$, with the beam contribution $\sim 5 \%$. The parameters for these target plasmas are listed in Table 3 .

(2) The neutral beam injection system has a power of $1.5 \mathrm{MW}$, an energy of $25 \mathrm{keV}$, and a beam current of order of $60 \mathrm{~A}[80]$. The corresponding beam density is $5 \times 10^{16} \mathrm{~m}^{-3}$, and the beam velocity is $2 \times 10^{6} \mathrm{~m} / \mathrm{s}$. This input power is expected to be sufficient to balance the energy loss from the FRC, as described in Sec. 4.2.

The neutral beam injection system [81] of $20 \mathrm{kV}$ $25 \mathrm{kV}$ and $60 \mathrm{~A}$, similar to the type presently used in the Madison Symmetric Torus facility at the University of Wisconsin, is assumed for these studies. The neutral beam consists primarily $(90 \%)$ of hydrogen or deuterium atoms.

It is important to minimize the beam spot size inside the plasma at $R=R_{\text {tang. }}$. With the half-angle divergence of 0.025 radians that has been demonstrated on the MST beam, the beam spot at the focal distance has a full width at the $1 / \mathrm{e}$ intensity point of $11 \mathrm{~cm}$. Assuming the usual Gaussian intensity profile, $50 \%$ of the power is deposited inside a diameter of $10 \mathrm{~cm}$ and $90 \%$ is deposited inside a diameter of $17 \mathrm{~cm}$. The expected density profile shape is centrally concentrated, and this is expected to localize the deposition near the tangency point.

The center-line of the neutral beam should lie in the midplane and be aimed near the major radius of the fast ion orbits with minimal radial excursions, $R_{\text {tang }} \sim 34 \mathrm{~cm}$, in order to minimize the fast ion loss caused by chargeexchange and collisions with structures just outside the plasma. The beam is well collimated in real space and velocity space, and very little pitch angle scattering occurs before the fast ions have been greatly slowed, so the calculated orbits avoid the ends of the configuration and closefitting structures in this area would probably not create significant losses.

Neutral beam deposition is calculated using a Monte Carlo algorithm to select rays with a Gaussian distribution representative of the beam spot size. The path integral for ionization of the incoming neutral hydrogen atoms along each ray is calculated, and the ionization locations are obtained by sampling from a decaying exponential distribution. This algorithm provides the starting positions for the orbit following simulation described later in this section. Ionization due to charge exchange dominates, but ionization from proton and electron impact is also included.

The radial excursion of fast ion orbits (and their motion out of the midplane) is fully modeled, using a source distribution calculated in the manner described above. The 'birth' distribution of newly ionized fast ions is shown in Fig. 11 as a function of major radius and as distance from the beam tangency point. The peak of the deposition occurs before the plasma density maximum at the tangency location. This indicates that the neutral beam is significantly attenuated as it moves into the plasma and that the beam would be more deeply deposited if the density were lower.

Full-orbit test particle simulations of the confinement of the neutral-beam-injected ions in an FRC configuration as shown in Fig. 12 have shown that, for injection energy of $E_{\mathrm{b}}=25 \mathrm{keV}$ and $B=1 \mathrm{kG}$, the relative number of particles lost by direct losses (collision with conducting shell) depends strongly on tangential radius of injection (Fig. 12 (top)). The initial deposition profiles for the different $R_{\text {tang }}$ have been calculated by the method mentioned above, and these were used as initial conditions for simulation runs. Results are shown in Fig. 12, where the number of confined particles is plotted versus the conducting shell radius for $R_{\mathrm{tang}}=34 \mathrm{~cm}$ (separatrix radius $R_{\mathrm{s}}=40 \mathrm{~cm}$ ). The direct losses of the neutral-beam-injected ions are smaller for larger $R_{\text {tang }}$ due to the smaller initial radial velocity of the NBI particles, and therefore the smaller radial extent of the 

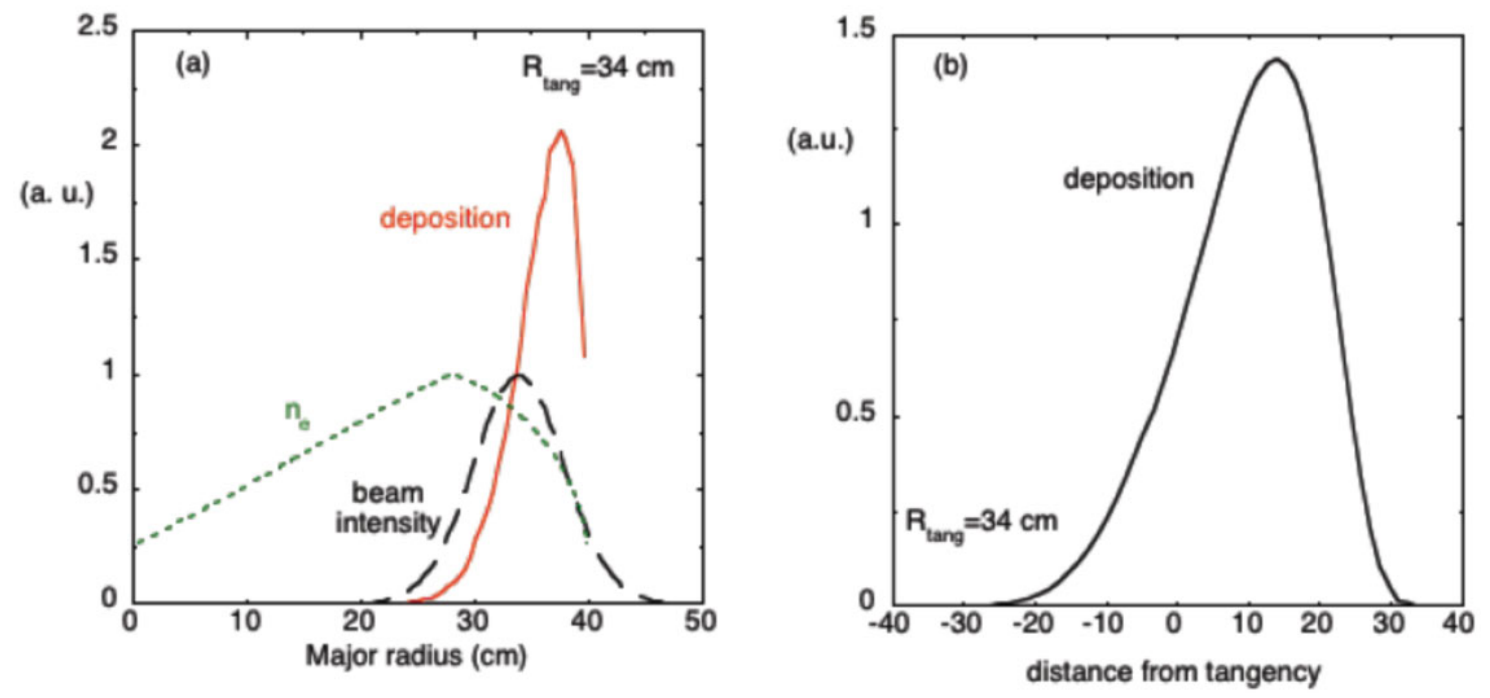

Fig. 11 (a) Plasma density, beam intensity, and deposition profile plotted versus R; (b) deposition profile plotted versus distance from the beam tangency location.



orbit [54]. For larger field $(B=1.5 \mathrm{kG})$, good confinement of the beam ions is achieved for a range of the injection radii as also shown in Fig. 12 (top).

\section{Summary}

This paper has described the goals, scientific foundations, scoping studies, and experimental details of the SPIRIT concept for oblate FRC research. This concept utilizes counter-helicity spheromak merging for the formation of large-flux FRCs. Conducting shells are utilized to stabilize $n=1$ tilt/shift instabilities. An ohmic solenoid is used to sustain the configuration and ramp-up the poloidal

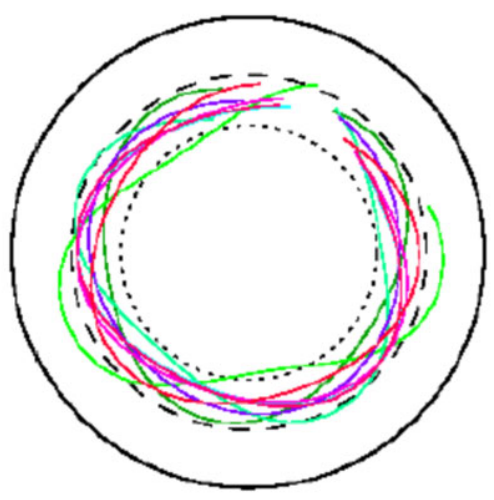

Fig. 12 (top) Number of confined NBI ions versus radial position of conducting shell for $R_{\mathrm{tang}}=34 \mathrm{~cm}$ and $E_{\mathrm{b}}=25 \mathrm{keV}$. The edge magnetic field is $B=1 \mathrm{kG}$ (red curve) and $B=$ $1.5 \mathrm{kG}$ (green curve). (bottom) Typical orbits of NBI ions for $R_{\mathrm{tang}}=34 \mathrm{~cm}, E_{\mathrm{b}}=25 \mathrm{keV}$, and $B=1 \mathrm{kG}$; dashed line shows the separatrix position.

flux. Neutral-beam injection is utilized to suppress cointerchange modes via FLR effects, as well as assisting in sustaining the configuration.

Theoretical scoping studies of the SPIRIT concept have developed a regime where the combination of oblate shape, conducting shells, current sustainment, and NBI lead to an FRC stable to all global MHD modes. Preliminary experimental scoping studies have demonstrated the formation of FRC plasmas in the MRX facility. Extensive orbit calculations illustrate that for experimentally achievable values of poloidal flux in MRX, the confinement of high energy ions from NBI should be acceptable for the physics goals of the experiment. The SPIRIT research program described here presents a promising, physics-based path for progress towards an attractive FRC reactor concept.

\section{Acknowledgments}

The authors thank Michiaki Inomoto for his help in the experimental scoping studies described above, and Stewart Zweben for help in developing and supporting the SPIRIT concept. This research has been funded by the US 
Department of Energy.

[1] “Compact Toroids," Special Issue, Fusion Tech. 9 (1986).

[2] M. Tuszewski, Nucl. Fusion 28, 2033 (1988).

[3] L.C. Steinhauer et al., Fusion Tech. 30, 116 (1996).

[4] M. Yamada, N. Pomphrey, Y. Ono and M. Katsurai, Nucl. Fusion 36, 1210 (1996).

[5] M. Katsurai and M. Yamada, Nucl. Fusion 22, 1407 (1982).

[6] M. Yamada, H. Ji, S. Hsu, T. Carter, R. Kulsrud, N. Bretz, F. Jobes, Y. Ono and F. Perkins, Phys. Plasmas 4, 1936 (1997).

[7] H. Momota, A. Ishida, Y. Kohzaki, G.H. Miley, S. Ohi, M. Ohnishi, K. Yoskikawa, K. Sato, L.C. Steinhauer, Y. Tomita and M. Tuszewski, Fusion Technol. 21, 2307 (1992).

[8] E.V. Belova, S.C. Jardin, H. Ji, M. Yamada and R. Kulsrud, Phys. Plasmas 7, 4996 (2000).

[9] E.V. Belova, S.C. Jardin, H. Ji, M. Yamada and R. Kulsrud, Phys. Plasmas 8, 1267 (2001).

[10] E.V. Belova, R.C. Davidson, H. Ji and M. Yamada, Phys. Plasmas 10, 2361 (2003).

[11] E.V. Belova, R.C. Davidson, H. Ji and M. Yamada, Phys. Plasmas 11, 2523 (2004)

[12] E.V. Belova, R.C. Davidson, H. Ji, M. Yamada, C.D. Cothran, M.R. Brown and M.J. Schaffer, Nucl. Fusion 46, 162 (2006)

[13] E.V. Belova, R.C. Davidson, H. Ji and M. Yamada, Phys. Plasmas 13, 056115 (2006).

[14] M. Yamada, Y. Ono, A. Hayakawa, M. Katsurai and F.W. Perkins, Phys. Rev. Lett. 65, 721 (1990)

[15] Y. Ono, A. Morita, T. Itagaki and M. Katsurai, Plasma Physics and Controlled Nuclear Fusion Research 1992 (Proc. 14th Int. Conf., Würzburg, Germany, 1992), Vol.2 (International Atomic Energy Agency, Vienna, 1993) p.619.

[16] Y. Ono, M. Yamada, T. Akao, T. Tajima and R. Matsumoto, Phys. Rev. Lett. 76, 3328 (1996).

[17] Y. Ono, M. Inomoto, Y. Ueda, T. Matsuyama and T. Okazaki, Nucl. Fusion 39, 2001 (1999).

[18] Y. Ono, T. Matsuyama, K. Umeda and E. Kawamori, Nucl. Fusion 43, 649 (2003).

[19] E. Kawamori and Y. Ono, Phys. Rev. Lett. 95, 085003 (2005)

[20] E. Kawamori, Y. Murata, K. Umeda, D. Hirota, T. Ogawa, T. Sumikawa, T. Iwama, K. Ishii, T. Kado, T. Itagaki, M. Katsurai, A. Balandin and Y. Ono, Nucl. Fusion 45, 843 (2005)

[21] E. Kawamori, T. Sumikawa, H. imanaka, R. Imazawa, K. Yamashita, T. Hayamizu, K. Umeda and Y. Ono, IAEA Fusion Energy Conference (Chengdu, China, 2006), Paper Ex-P7-13.

[22] C.D. Cothran, A. Falk, A. Fefferman, M. Landreman, M.R. Brown and M.J. Shaffer, Phys. Plasmas 10, 1748 (2003).

[23] M. Yamada, H. Ji, T.A. Carter, S.C. Hsu, R.M. Kulsrud, N.L. Bretz, F.C. Jobes, Y. Ono, M. Katsurai, T.-H. Watanabe, T. Sato and T. Hayashi, Fusion Energy 1996 (Proc. 16th Int. Conf., Montreal, Canada, 1996), Paper IAEA-CN-64/CP-19.

[24] S.P. Gerhardt, E. Belova, M. Inomoto, M. Yamada, H. Ji, Y. Ren and A. Kuritsyn, Phys. Plasmas 13, 112508 (2006); H. Ji, E. Belova, S.P. Gerhardt and M. Yamada, J. Fusion Energy, 10.1007/_s10894-006-9043-4 (2006).

[25] M.N. Rosenbluth and M.N. Bussac, Nucl. Fusion 19, 489 (1979)

[26] J.H. Hammer, Nucl. Fusion 21, 488 (1981).
[27] R.D. Milroy, D.C. Barnes, R.C. Bishop and R.B. Webster, Phys. Plasmas B 1, 1225 (1989).

[28] W.A. Newcomb, Phys. Fluids 23, 2296 (1980).

[29] J.R. Cary, Phys. Fluids 24, 2239 (1981).

[30] A. Ishida, N. Shibata and L.C. Steinhauer, Phys. Plasmas 1, 4022 (1994).

[31] S. Ohi, T. Minato, Y. Kawakami, M. Tanjyo, S. Okada, Y. Ito, M. Kako, S. Goto, T. Ishimura and H. Ito, Phys. Rev. Lett. 51, 1042 (1983).

[32] H.Y. Guo, A.L. Hoffman, K.E. Miller and L.C. Steinhauer, Phys. Rev. Lett. 92, 245001 (2004).

[33] J.T. Slough and K.E. Miller, Phys. Rev. Lett. 85, 1444 (2000).

[34] H.Y. Guo, A.L. Hoffman, R.D. Brooks, A.M. Peter, Z.A. Pietrzyk, S.J. Tobin and G.R. Votroubek, Phys. Plasmas 9, 185 (2002).

[35] H.Y. Guo, A.L. Hoffman, R.D. Milroy, K.E. Miller and G.R. Votroubek, Phys. Rev. Lett. 94, 185001 (2005).

[36] T. Intrator, S.Y. Zhang, J.H Degnan, I. Furno, C. Grabowski, S.C. Hsu, E.L. Ruden, P.G. Sanchez, J.M. Taccetti, M. Tuszewski, W.J. Waganaar and G.A. Wurden, Phys. Plasmas 11, 2580 (2004).

[37] S. Zhang, T. Intrator, G.A. Wurden, W.J. Waganaar, J.M. Taccetti, R. Renneke, C. Grabowski and E.L. Ruden, Phys. Plasmas 12, 052513 (2005).

[38] M. Yamada, H. Ji and P. Heitzenroeder, Proc. ICC, "Innovative Approaches to Fusion Energy" Pleasanton, Calif. Oct. (1997).

[39] M. Yamada and H. Ji, J. Plasma Fusion Res. Ser. 2, 62-66 (1999), H. Ji and M. Yamada, J. Plasma Fusin Res. SERIES 2, 195-197.

[40] M. Tuszewski, D.C. Barnes, R.E. Chrien, J.W. Cobb, D.J. Rej, R.E. Siemon, D.P. Taggart and B.L. Wright, Phys. Rev. Lett. 66, 711 (1991).

[41] A. Ishida, H. Momota and L.C. Steinhauer, Phys. Fluids 31, 3024 (1988).

[42] N. Iwasawa, A. Ishida and L.C. Steinhauer, Phys. Plasmas 8, 1240 (2001).

[43] E.V. Belova et al., Plasma Physics and Controlled Nuclear Fusion Research, Proc. the 18th Int. Conf., IAEA (2000), paper IAEA-CN-77/THP2/02.

[44] D.C. Barnes, Phys. Plasmas 8, 4856 (2001).

[45] D.C. Barnes, Phys. Plasmas 9, 560 (2002).

[46] H. Yamada, T. Katano, A. Ishida and L.C. Steinhauer, Phys. Plasmas 10, 1168 (2003).

[47] H. Ohtani, R. Horiuchi and T. Sato, Phys. Plasmas 10, 145 (2003).

[48] L.C. Steinhauer and A. Ishida, Phys. Rev. Lett. 79, 3423 (1997).

[49] L.C. Steinhauer, H. Yamada and A. Ishida, Phys. Plasmas 8, 4053 (2001).

[50] L.C. Steinhauer, Phys. Plasmas 9, 3767 (2002).

[51] S.M. Mahajan and Z. Yoshida, Phys. Rev. Lett. 81, 4863 (1998).

[52] Z. Yoshida, S.M. Mahajan, S. Ohkasi, M. Iqbal and N. Shatashvilli, Phys. Plasmas 8, 2125 (2001).

[53] T. Takahashi, Y. Tomita, H. Momota and N.V. Shabrov, Phys. Plasmas 4, 4301 (1997).

[54] A.F. Lifschitz, R. Farengo and A.L. Hoffman, Nucl. Fusion 44, 1015 (2004).

[55] A.F. Lifschitz, R. Farengo and N.R. Arista, Nucl. Fusion 42, 863 (2002)

[56] M. Tuszewski, D.P. Taggart, R.E. Chrien, D.J. Rej, R.E. Siemon and B.L. Wright, Phys. Fluids B 3, 2856 (1991). 
[57] J.M. Finn and R.N. Sudan, Nucl. Fusion 22, 1443 (1982); J.M. Finn, Phys. Fluids 24, 274 (1981).

[58] D.C. Barnes and R.D. Milroy, Phys. Fluids B 3, 2609 (1991).

[59] R.N. Sudan and M.N. Rosenbluth, Phys. Fluids 22, 282 (1979).

[60] R.V. Lovelace, Phys. Fluids 19, 723 (1976).

[61] H. Ji, M. Yamada, R. Kulsrud, N. Pomphrey and H. Hihura, Phys. Plasmas 5, 3685 (1998).

[62] I.E. Jones and W.N. Hugrass, J. Plasma Phys. 26, 441 (1981).

[63] T. Asai, M. Inomoto, N. Iwasawa, S. Okada and S. Goto, Phys. Plasmas 10, 3608 (2003).

[64] T. Asai, Y. Suzuki, T. Yoneda, F. Kodera, M. Okubo, S. Okada and S. Goto, Phys. Plasmas 7, 2294 (2000).

[65] S. Okada, T. Asai, F. Odera, K. Kitano, Y. Suzuki, K. Yamanaka, T. Kanki, M. Inomoto, S. Yoshimura, M. Okubo, S. Sugimoto, S. Ohi and S. Goto, Nucl. Fusion 41, 625 (2001).

[66] A.L. Hoffman, Phys. Plasmas 5, 979 (1998).

[67] R.D. Milroy, Phys. Plasmas 6, 2771 (1999).

[68] S.A. Cohen and R.D. Milroy, Phys. Plasmas 7, 2539 (2000).

[69] J.H. Hammer and H.L. Berk, Nucl. Fusion 22, 89 (1982).

[70] N. Rostoker, M.W. Binderbauer and H.J. Monkhorst, Sci- ence 278, 1419 (1997).

[71] J.T. Slough and K.E. Miller, Phys. Plasmas 7, 1945 (2000).

[72] A.L. Hoffman, H.Y. Guo, R.D. Milroy and Z.A. Pietrzyk, Nucl. Fusion 43, 1091 (2003).

[73] R.D. Milroy and K.E. Miller, Phys. Plasmas 11, 633 (2004).

[74] J.F. Santarius and J.D. Callen, Phys. Fluids 26, 1037 (1983).

[75] H.L. Berk, H. Momota and T. Tajima, Phys. Fluids 30, 3548 (1987).

[76] The probe, which uses optical fibers to collect light from a small volume for Doppler spectroscopy, is similar to that described in G. Fiksel, D.J. Den Hartog, and P.W. Fontana, Rev. Sci. Instrum. 69, 2024 (1998).

[77] M. Yamada, H.P. Furth, W. Hsu, A. Janos, S. Jardin, M. Okabayashi, J. Sinnis, T.H. Stix and K. Yamazaki, Phys. Rev. Lett. 46, 188 (1981).

[78] P.E. Young, F.J. Wysocki, M. Yamada and A. Janos, Phys. Fluids B 3, 2591 (1991).

[79] A.L. Hoffman and J.T. Slough, Nucl. Fusion 33, 27 (1993).

[80] A.B. Hassam, R. M. Kulsrud, R. J. Goldston, H. Ji and M. Yamada, Phys. Rev. Lett. 83, 2969 (1999).

[81] P.P. Deichuli, V.I. Davydenko, A.A. ivanov, S.A. Korenpanov, V.V. Mishagin, A.V. Sorokin, N.V. Stupishin and G.I. Shulzhenko, Rev. Sci. Instr. 75, 1816 (2004). 